\title{
Criterios \\ El Palacio de San Telmo en el siglo XIX
}

Vicente Lleó Cañal

Catedrático de Historia del Arte. Universidad de Sevilla

\section{Resumen}

El presente texto trata de analizar especialmente la Intervención del arquitecto Balbino Marrón para transformar el Seminario en Palacio, coincidiendo con la llegada de los duques de Montpensier a la ciudad de Sevilla, en la segunda mitad del siglo XIX. La aportación de Marrón confiere al edificio una unidad definitiva, convirtiéndolo en un recinto acabado y cerrado sobre sí mismo. Igualmente, el arquitecto supo ejecutar con gran sensibilidad la rehabilitación como palacio, con las modificaciones funcionales que la transformación implicaba.

\section{Palabras clave}

\section{Palacio de San Telmo}

Sevilla

Siglo XIX

Historia

Patrimonio inmueble

Fuentes documentales
Al llegar a Sevilla los Duques de Montpensier en 1848, se instalaron provisionalmente en el Palacio Arzobispal, mientras que se terminaban las obras de acondicionamiento en el Alcázar; allí nacerá su hija mayor la Infanta Isabel (21-9-1848). Pero enseguida empezaría a hacer gestiones para comprar al Estado el Colegio de San Telmo que, habiendo cesado de funcionar como Colegio de Náutica desde 1847, tuvo usos dispares como sede de la Sociedad del Ferrocarril y de la Universidad Literaria, encontrándose infrautilizado y con sus obras paralizadas.

Para la valoración del edificio, en 1849, intervinieron, por parte del Rector de la Universidad de Sevilla (el edificio había pasado a depender del Ministerio de Instrucción Pública) el arquitecto Juan Manuel Caballero; por parte de Montpensier el arquitecto Balbino Marrón (+ 1867), quien va a ser el encargado de la rehabilitación del Colegio como Palacio. El edificio con sus terrenos estimados en 17.790 vs.2 fue valorado en 1.504 .800 reales, a lo que habría que añadir las minuciosas valoraciones de todos los bienes muebles y obras de arte (las pinturas fueron tasadas por Joaquín Domínguez Bécquer) pero incluyendo hasta los objetos de la carpintería.

Para entender la operación de San Telmo, hay que tener en cuenta que la aspiración de Montpensier era sustituir a su cuñada Isabel II. Como es bien sabido ese había sido el plan urdido por el Rey Luis Felipe de Francia quien, confiado en los informes diplomáticos que hablaban de la mala salud de Isabel II, decidió casar a su hijo Antonio con la Princesa de Asturias como vía indirecta para alcanzar el trono. Este objetivo, repetidamente frustrado no impidió que participase en numerosas conspiraciones y que contribuyese económicamente a la Revolución de 1868. El palacio sevillano vino a adquirir así un valor simbólico, como corte "alternativa" a la madrileña, un palacio moderno en los aspectos técnicos (instalación de electricidad. de telégrafo, cuartos de baño, etc.) pero respetuoso con las tradiciones españolas tanto históricas (series de retratos de personalidades españolas) como artísticas (potenciando el barroco casticista de Figueroa) e incluso locales (acudiendo casi exclusivamente a artistas y artesanos locales para las reformas). Es importante señalar la voluntad de Montpensier porque, aunque la ejecución de las obras correspondió a Marrón, la última palabra, incluso en los aspectos más nimios correspondió siempre al Infante. Una muestra de ello nos lo ofrece un dibujo de Marrón, de fecha imprecisa, pero de 1852-3 probablemente que muestra dos alternativas de diseño para la portada de piedra del Salón de las Columnas; las notas del arquitecto señalan "en el anta A se han establecido las orlas 0 de mármol blanco, como así S.A.S. Io mandara sin haber hecho otra modificación en el repartimiento de sus recuadros, pues están en todo conformes a los que se expresaban en el diseño anterior. En el anta B se observa una modificación general en la simetría de los recuadros, la que hemos puesto por si agradase más a S.A.S". Incluso cuando estaba fuera de Sevilla, el control de Montpensier sobre el diseño del palacio fue absoluto: la correspondencia cruzada entre el secretario del Duque, Joaquín 


\section{$072-073$ Criterios}

El Palacio de San Telmo en el siglo XIX

\section{PH51 - Diciembre 2004}

del Alcázar, que lo había acompañado a Madrid en 1857, y su intendente en Sevilla Isidoro de las Cagigas, revela que absolutamente nada escapaba a su decisión.

Para sus fines, Montpensier contó con un auxiliar imprescindible: Balbino Marrón, con una capacidad de trabajo equivalente a la de su patrono. Hay que tener en cuenta que en los años 50, al mismo tiempo que Marrón se ocupa de San Telmo, está también dedicado a la creación de los palacios de Villamanrique de la Condesa, Castilleja y Sanlúcar de Barrameda y a los caseríos de las Haciendas de Gambogaz, San Luis, del Vado y Majaloba, entre otros proyectos.

Las rehabilitación del Colegio de San Telmo como Palacio ocuparía a Balbino Marrón, hasta su muerte en 1867; la abundantísima documentación del Archivo Orleans de Sanlúcar no deja lugar a dudas de que a él se debió toda la obra principal, apareciendo Talavera en época muy posterior y sólo como decorador o como autor de las desdichadas obras de adaptación del edificio con posterioridad a la enajenación de el Convento de San Diego, que acabaron con unos de los espacios más solemnes del palacio: el Apeadero.

El plano más antiguo de los conservados, obra de Marrón (2), es de 1849 y muestra el estado del edificio tal como se encontraba al adquirirlo Montpensier; debió hacerse a efectos de la tasación pues lleva anotaciones a lápiz de mediciones. Lo más notable de este plano es:

$>$ En el lado S. aparecen adosadas al viejo dormitorio una serie de edificaciones antiguas, seguramente anteriores a la propia construcción del Seminario, que nunca llegaron a demolerse.

$>$ En el lado E. sobresale la cabecera de la capilla; en el lado SE el frente se cierra por un conjunto de patios, corrales y dependencias correspondientes básicamente a las que se ven en el plano de 1781. Al NE de la cabecera aparece el solar establecido por los planos originales del edificio todavía sin construir, aunque con las zanjas de la cimentación abiertas.

$>$ La fachada N. y la torre NE aparecen aún sin construir, tan sólo abiertas de cimientos.

> La disposición interna del sector S. se muestra sustancialmente igual a la que refleja el plano de 1781; sólo que el llamado en los documentos "dormitorio nuevo", que formaba ángulo con el viejo y mira a los jardines, aparece ahora tabicado y dividido en cámaras individuales (¿una reforma quizás para alojar a los porcionistas que pagaban?).

Poco posterior, quizás de 1850 , es un nuevo plano del palacio. Éste no muestra diferencias notables con él (2). Tan sólo se han limpiado de las edificaciones adosadas los frentes S. y E., mientras que la nave del Ilamado dormitorio nuevo, frente a los jar- dines, aparece otra vez sin tabiques y con las columnas dispuestas en el centro. Los frentes S. y E., una vez limpios de adosados, muestran una distribución de huecos al exterior totalmente irregular, fruto evidentemente de no estar pensadas originalmente como fachadas exentas. En la fachada principal, finalmente, se restauró la portada de piedra, afectada por un rayo en 1836, colocándose el remate con las armas de Montpensier entre esculturas alegóricas De esta obra se conserva el presupuesto firmado por el cantero José Barrada y por el maestro de obras José Gutiérrez el 22 de agosto de 1850. También del mismo años es el contrato firmado con Antonio Cabral Bejarano para la decoración de la Capilla, que incluyó la abertura de una tribuna privada e importantes obras de pintura, dorado y mobiliario litúrgico.

En alguna fecha anterior a 1857 (que es la del plano 4b), quizás 1852, Marrón diseñó las nuevas fachadas S. y E., incluida la torre en el ángulo (4), que se realizan contemporáneamente con importantes transformaciones interiores:

> Quizás la más importante fue la creación del Ilamado Salón de las Columnas en el primitivo dormitorio del seminario. Marrón demolió la primitiva fachada y la sustituyó por una arquería sobre columnas pareadas, las mismas que habían estado dispuestas a lo largo de la antigua nave; en el centro de la fachada levantó una portada de mármoles de colores flanqueada por estípites, en homenaje al barroco de Figueroa y rematada por balcón con las estatuas de San Fernando y San Luis. Este salón se hizo de una sola altura, rematado por azotea entre las dos torres. En fechas posteriores se realizó la decoración interior: en 1857 se estaba solando de mármol y colocando mesas del mismo material- Aquí también, en fecha difícil de precisar, se instalaron los techos pintados por Tegeo, procedentes del Palacio madrileño de Buenavista que habían sido heredados por la Infanta María Luisa. En los extremos de este salón se dispusieron unos gabinetes y en la misma planta, en las torres de las esquinas techos abovedados sostenidos por columnas centrales.

$>$ El frente E., una vez demolidos los antiguos corrales y hasta la cabecera de la capilla, fue dotado de una nueva fachada inspirada en la delantera de San Telmo pero también en otras obras de Leonardo de Figueroa (San Pablo, Venerables, etc.), con fabrica de ladrillo en dos colores y decoraciones cerámicas. También, para solucionar los desniveles. se levantó una terraza delante Como veremos este frente fue continuado en épocas posteriores en dirección N. También a esta época se debe la regularización de los huecos, cuyos enmarcamientos Ilevan la flor de lis de los Orleans.

$>$ El frente N. aparece, como dijimos, cerrado, según el proyecto primitivo y los cimientos abiertos, con una nueva torre en el ángulo NE: la crujía de fachada entre estas torres muestra una portada en el centro diseñada por Marrón, que da acceso a un amplio espacio abierto con la función de Patio Apeadero. El frente a 
1. Planta del Colegio Seminario para huérfanos de marineros, que se proyecta fundar en Sevilla (1681). Ministerio de Cultura. Archivo General de Indias. MP. Europa y África, 13

2. Balbino Marrón, planta del estado del palacio en el momento de su adquisición
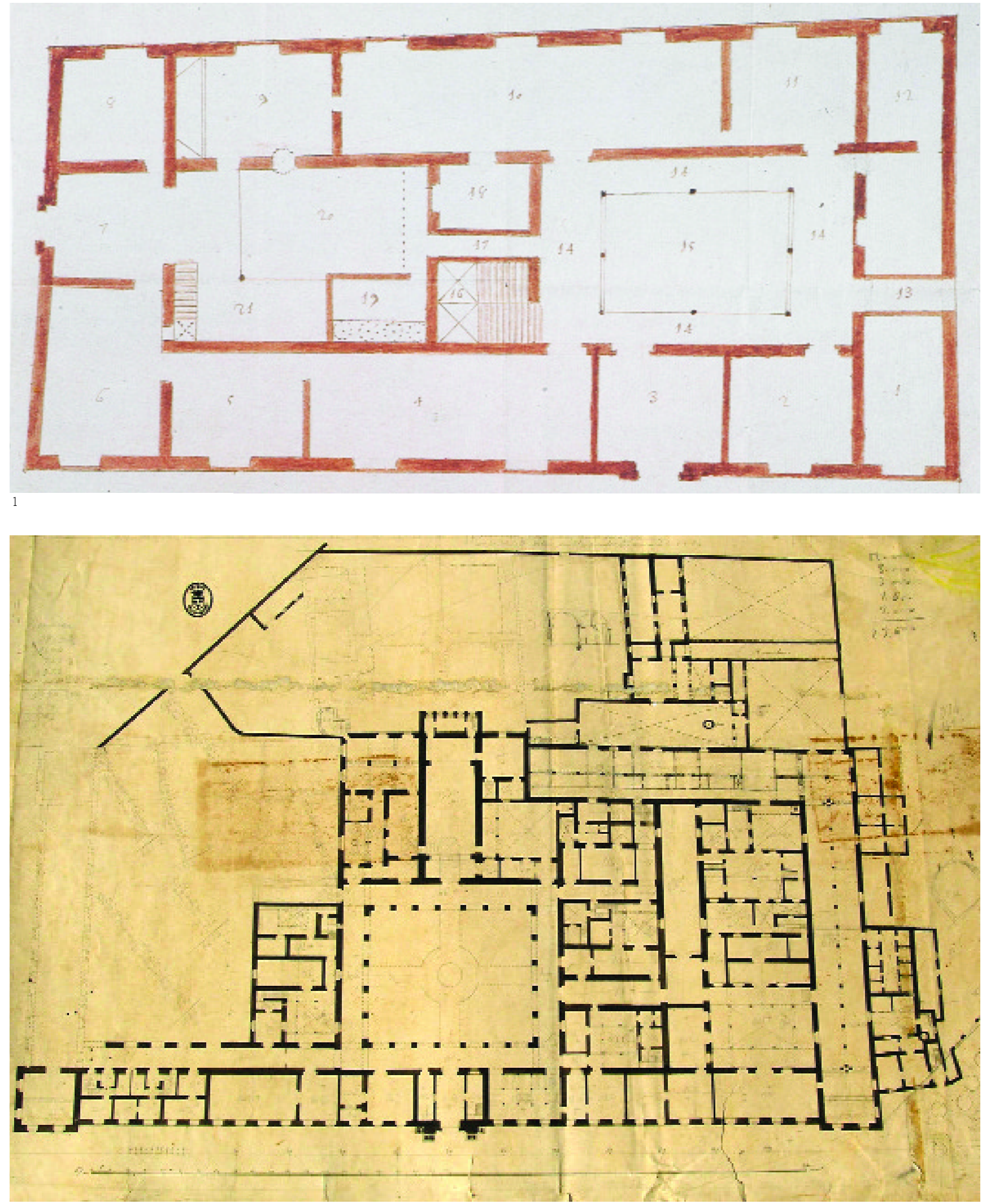


\section{$074-075$ \\ Criterios \\ El Palacio de San Telmo \\ en el siglo XIX \\ PH51 - Diciembre 2004}

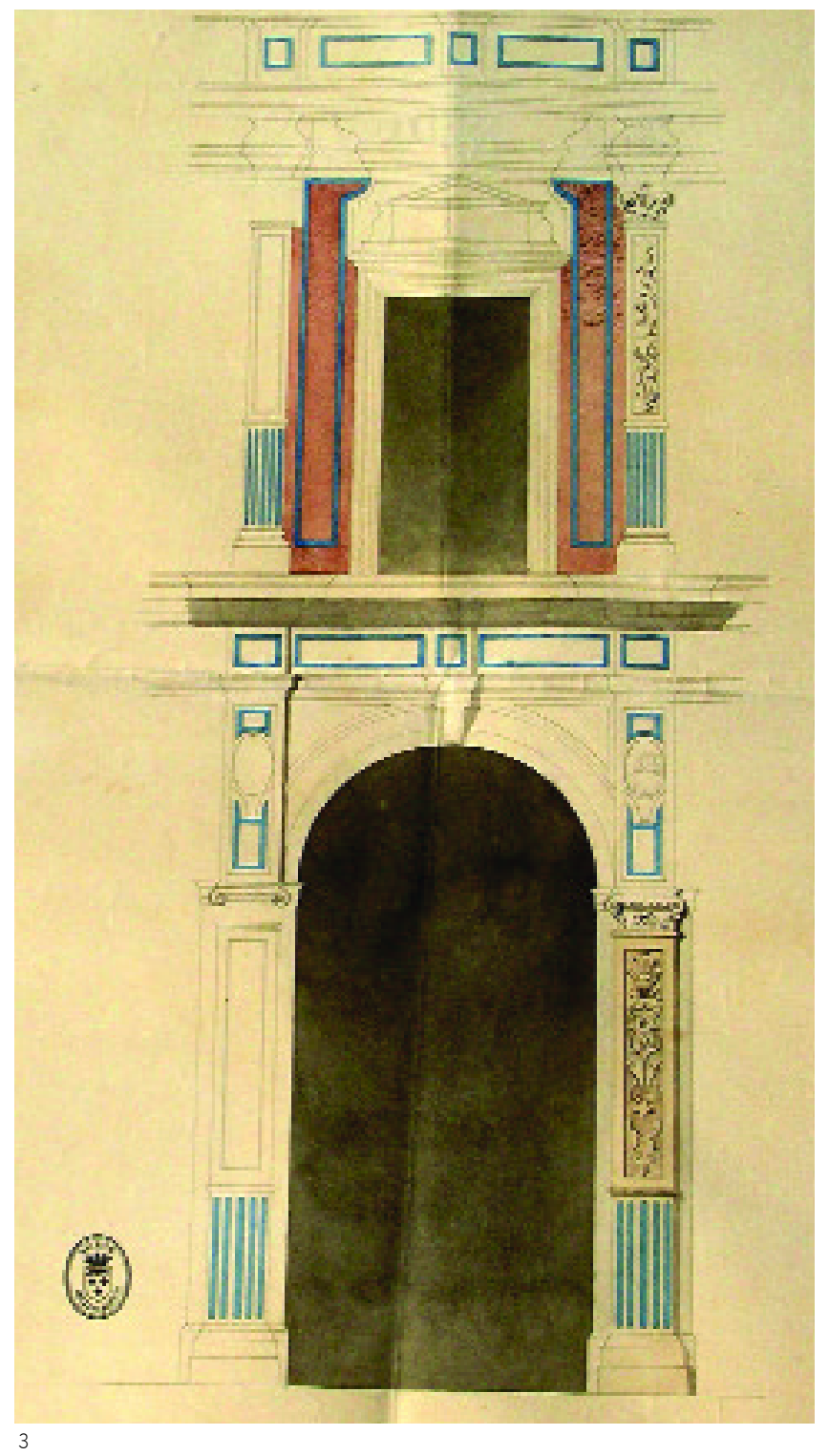

los jardines quedó cerrado provisionalmente a la izquierda del ábside de la capilla por una simple tapia.

> Posiblemente también por estas fechas Marrón reformó el patio principal. Cintora había eliminado la decoración de grutescos de las pilastras y enjutas del proyecto de Figueroa (que consideraba "impertinentes")con el pretexto de la economía, pues quedaban tres pandas por concluir, estando terminada sólo la de delante de la Capilla. Marrón la va a reponer siguiendo posiblemente los dibujos originales de Figueroa.

> Otras modificaciones menores son detectables en el antiguo patio de cocinas, refectorio (eliminación de tabiques), patio $\mathrm{n}^{0} 7$ del plano de 1781 (que pasa de 5 a 7 columnas) y crujía de la fachada principal (eliminación de tabiques)
3. Balbino Marrón, alzado de un tramo del patio principal mostrando las soluciones de Figueroa y Cintora (c. 1855). Archivo Fundación Infantes Duques de Montpensier

4. Balbino Marrón, proyecto de nueva fachada entre la torre SE y la cabecera de la capilla (c. 1852). Archivo Fundación Infantes Duques de Montpensier

El plano siguiente muestra una situación parecida a la que acabamos de describir pero tiene la ventaja de estar fechado: 16 de agosto de 1857; contiene el esquema de distribución de aguas.

Hacia 1860 debe fecharse el plano siguiente que muestra importantes novedades. En primer lugar aparece ya la verja de hierro levantada en los frentes 0 . y $\mathrm{N}$. que es la misma existente en la actualidad; en realidad, esta verja estaba prevista ya desde 1850 , según trazas de Juan Lizasoain quien en su memoria establece como básico "que no se pierdan los ejes principales de la verja por falta de acuerdo con los de la fachada".

En el mismo plano aparece también una amplia edificación situada en el límite E. del Patio Apeadero: es el picadero que lleva en su extremo unas estancias auxiliares, cuya función no se especifica. En el frente $\mathrm{S}$. de este patio apeadero encontramos unas nuevas construcciones destinadas a resolver un problema compositivo que generó un notable volumen de documentación.

En planos anteriores, en el frente 0 . del apeadero se puede ver una especie de pórtico sobre columnas que estaba destinado a recibir a los visitantes. En efecto, aunque la fachada principal siguió siendo la trazada. por Leonardo de Figueroa, la N. se convirtió en la entrada principal o de carruajes, de modo que los que entraban por la puerta nueva trazada por Marrón (que contenía el cuerpo de guardia) tenían que girar a la derecha para depositar a sus ocupantes a cubierto bajo este pórtico. Pero el acceso al palacio por este pórtico desembocaba en un corredor largo, estrecho y poco iluminado, paralelo a la fachada. Marrón se propuso trazar una nueva entrada de mayor importancia en el frente $\mathrm{S}$. del Apeadero, con pórtico y fachada más elaborados y probablemente una nueva escalera con iluminación cenital; se conservan numerosos dibujos con distintas soluciones para este nuevo acceso pero aparentemente ninguna llegó a ejecutarse, así, en unos presupuestos fechados en 1860, Marrón revela que las obras estaban "suspendidas desde hace tiempo". Los problemas eran evidentes: en primer lugar era imposible centrar la portada del nuevo apeadero en la fachada y al mismo tiempo alinearla a eje con la portada de la calle y, en segundo lugar, aunque desembocaba antes en el patio principal que con el apeadero antiguo, tampoco solucionaba las tortuosas comunicaciones con espacios importantes como el Salón de las Columnas. Los proyectos sucesivos para esta pieza, tanto en planta como en alzado llegan hasta el año de 1864.

Finalmente, la fachada exterior del nuevo picadero que cerraba el lado N. de la capilla así como la torre del ángulo recibieron el mismo tratamiento que las anteriores, con sus huecos regularizados, fabrica de ladrillo en dos colores e incrustaciones de cerámica vidriada, además de una serie de relieves en la cabecera de la capilla.

Todas estos proyectos para el Patio del Apeadero, frustrados mayoritariamente y, en cualquier caso destruidos por intervenciones 


076 - 077
Criterios
El Palacio de San Telmo
en el siglo XIX
PH51 - Diciembre 2004

5. Balbino Marrón, proyecto de arreglo del sector Norte con patio apeadero y picadero (c. 1855). Archivo Fundación Infantes Duques de Montpensier

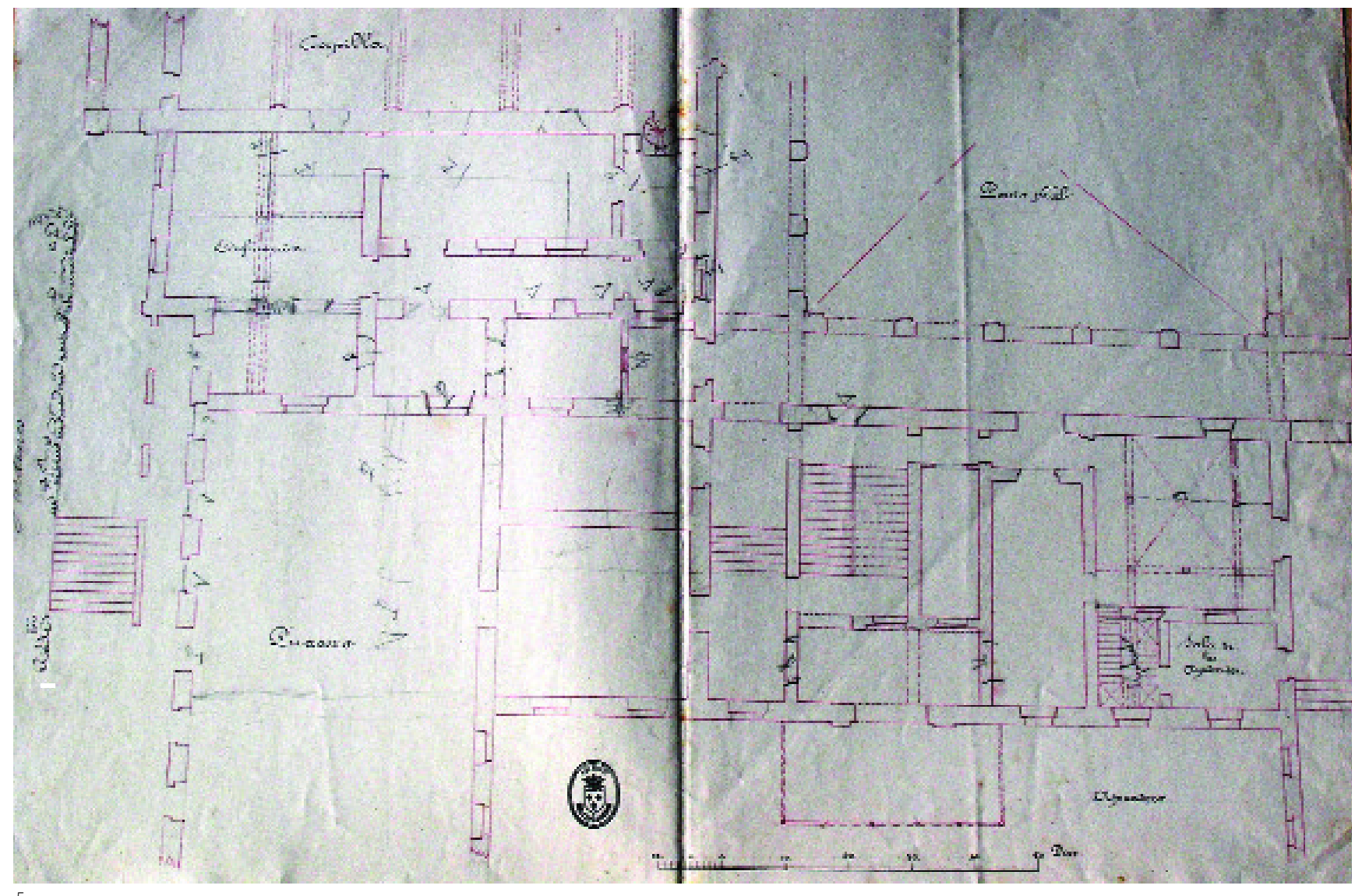

5

\section{Fondos documentales}

Archivo Histórico de la Universidad de Sevilla

Libros de Acuerdos de la Universidad de Mareantes, vols. 309, 310, 311, 312, 314

Libros de Cuenta y Razón de lo que se ha gastado en la Obra de la fábrica de San Telmo, vols. 207, 209

\section{Archivo de Protocolos Notariales de Sevilla} Legajos 5.152, 5.185, 11.108 y 11.110

\section{Archivo General de Indias}

Sección Indiferentes., legajos 1635 1636m 1637, 1639, 443, 444

Sección Contaduría, legajo 251

Sección Contratación, legajos 249 y 5786

Sección Arribadas, legajo 555

Archivo General de la Marina "Alvaro de Bazán" Sección Colegios. Colegio de San Telmo, legajos 983, 989m 990, 991, 992, 994, 995, 997
Archivo de la Fundación “Infantes Duques de Montpensier"

Caja 521. Estados de Obras para los años 1849-54 Carpetas 1, 2, 6

Caja 519. Correspondencia 1857

\section{Otras fuentes manuscritas}

Magán, N.V. "Historia del Palacio de San Telmo, propiedad y residencia actual de SS.AA.RR. Ios Serenisssimos Sres. Infantes, Duques de Montpensier" (1851. Biblioteca Excmo. Sr. Duque de Segorbe)

Anónimo. "Breve resúmen histórico de la fundación, progresos y actual estado del Colegio Nacional de San Telmo, extramuros de la Ciudad de Sevilla" (1846. Archivo Fundación Infantes Duques de Montpensier" 